\title{
The Inflammatory Bowel Disease-Fatigue Patient self-assessment scale: translation, cross-cultural adaptation and psychometric properties of the Brazilian version (IBD-F Brazil)
}

\author{
Ana Cristina LAGE*1, Cristino Carneiro OLIVEIRA ${ }^{1}$, Ana Paula Delgado Bomtempo BATALHA ${ }^{1}$, \\ Adaliza Furtado ARAÚJO², Wladyslawa CZUBER-DOCHAN ${ }^{3}$, Julio Maria Fonseca CHEBLI², \\ Laura Alves CABRAL ${ }^{1}$ and Carla MALAGUTI ${ }^{1}$
}

Received: 18 September 2019 Accepted: 21 November 2019

\begin{abstract}
Background - Fatigue is a common symptom in patients with inflammatory bowel diseases (IBD). A translated and culturally adapted, instrument with robust psychometric for measuring fatigue in Brazilian patients with IBD is needed. Objective - To translate and cross-culturally adapt the inflammatory Bowel Disease Fatigue Scale (IBD-F) into Brazilian-Portuguese and to test its measurement properties in Brazilian patients with IBD. Methods - Data from 123 patients with IBD were collected. In addition to IBD-F, the Functional Assessment of Chronic Illness Therapy-Fatigue (FACIT-F) was used. The measurement properties tested were: internal consistency, reproducibility (reliability and agreement), construct validity, internal and external responsiveness, and ceiling and floor effects. Results - The Brazilian-Portuguese version of the IBD-F showed excellent internal consistency (Cronbach's alpha of 0.95), excellent reproducibility (ICC $=0.97$ ) and a minimal detectable change of 6.0 points. The construct validity was demonstrated with a good correlation between the IBD-F and FACIT-F ( $\mathrm{r}=-0.46)$. Effect sizes used for measuring internal responsiveness were moderate among those with Crohn's (0.66) disease and low in patients with ulcerative colitis (0.24). The Brazilian-Portuguese version of the IBD-F presented with high external responsiveness for Crohn's disease (0.84) and with low external responsiveness for ulcerative colitis (0.33). The area under the curve considered for responsiveness was 0.84 . Twenty-five percent of floor effects and no ceiling effect were recorded. Conclusion The Brazilian-Portuguese version of IBD-F has adequate measurement properties and its use can be recommended in clinical practice and research. HEADINGS - Inflammatory bowel diseases. Reproducibility of results. Fatigue.
\end{abstract}

\section{INTRODUCTION}

Inflammatory bowel diseases (IBD) are idiopathic multisystemic disorders that present with periods of active and quiescent disease throughout its clinical course ${ }^{(1,2)}$. While ulcerative colitis is characterized by inflammation limited to the colonic mucosa, in Crohn's disease transmural inflammation can affect any part of the gastrointestinal tract ${ }^{(3)}$. In addition to intestinal symptoms, systemic manifestations are common among patients with IBD. General fatigue is a prevalent symptom affecting about $40 \%$ of patients in remission and also in about $86 \%$ in the active phase of the disease ${ }^{(4)}$. Fatigue is defined as a feeling of exhaustion, lack of energy, debilitating and of prolonged duration, affecting functional capacity and performance in activities of daily living ${ }^{(5,6)}$. In the IBD diseases, fatigue has been show to be associated with significant impairment in physical function and health-related quality of life $\mathrm{e}^{(7,8)}$.

In patients with IBD, fatigue has been measured using several scales mostly adapted from those used in chronic diseases trying to determine the patient's perception and the severity of this symptom $^{(9)}$. A recent systematic review identified nine different symptom assessment scales used in patients with IBD. The Multidimensional Fatigue Inventory and the Fatigue Impact Scale were the most frequently used instruments ${ }^{(9)}$, however, the Functional Assessment of Chronic Illness Therapy-Fatigue subscale (FACIT$\mathrm{F})$ is the only measure validated for use in patients with $\operatorname{IBD}^{(10)}$. In addition, the FACIT-F was initially developed to assess the functional consequences of fatigue in cancer patients, not specific for those with IBD.

The Inflammatory Bowel Disease Fatigue (IBD-F) scale was recently developed to evaluate the experience and effects of fatigue in patients with IBD ${ }^{(11)}$. The IBD-F measures fatigue symptom considering its most relevant aspects, severity and physical consequences and has robust psychometric properties ${ }^{(12,13,14,15)}$. The prevalence of IBD among Brazilians has been about 0.6 to 6.75 cases/100,000 inhabitants with Crohn's disease and 2.42 to 21 cases/100,000 inhabitants with ulcerative colitis ${ }^{(16)}$, and an appropriate evaluation of fatigue is crucial to guide better management and intervention for these patients. The IBD-F questionnaire may contribute to the

Declared conflict of interest of all authors: none

Disclosure of funding: this study was financed in part by the Coordenação de Aperfeiçoamento de Pessoal de Nível Superior - Brasil (CAPES) - Finance Code 001.

* in memoriam. ${ }^{1}$ Universidade Federal de Juiz de Fora, Programa de Pós-graduação em Ciências da Reabilitação e Desempenho Físico-Funcional, MG, Brasil. ${ }^{2}$ Universidade Federal de Juiz de Fora, Departamento de Medicina, Centro de Doenças Inflamatórias Intestinais, Hospital Universitário, MG, Brasil. ${ }^{3}$ Florence Nightingale School of Nursing \& Midwifery, King's College London, London, United Kingdom.

Corresponding author: Carla Malaguti, PhD. E-mail: carlamalaguti@gmail.com 
systematic evaluation of fatigue and help to understand its impact on the daily activities of patients with IBD $^{(11)}$. The aims of this study were (1) to translate and cross-culturally adapt the IBD-F scale in to Brazilian-Portuguese and (2) to test its measurement properties including internal consistency, reproducibility, construct validity, internal and external responsiveness and ceiling and floor effects in Brazilian patients with IBD.

\section{METHODS}

This study was performed in two phases. In Phase I the translation and cross-cultural adaptation of the IBD-F into BrazilianPortuguese were conducted (IBD-F Brazil). In Phase II the measurement properties of the IBD-F Brazil were explored. Phase I was conducted according to published guidelines for translation and cross-cultural adaptation of health-related questionnaires ${ }^{(17)}$. Phase II applied the quality criteria used for measurement properties of questionnaires ${ }^{(18)}$. The Consensus-based Standards for selection of health status Measurement Instruments (COSMIN) was used as guidance to inform about each measurement property reported $^{(19)}$. An authorization from the author of the IBD-F scale original version was obtained in advance. This study was approved by Human Ethic Committee at Federal University of Juiz de Fora, Minas Gerais, Brazil with the number 2.372.106 (CAAE 68473417.0.0000.5133).

This study was performed at Department of Cardiorespiratory and Musculoskeletal Physiotherapy, Faculty of Physiotherapy, UFJF and at Department of Medicine - Inflammatory Bowel Diseases Center, University Hospital, UFJF.

\section{Phase I - translation and cross-cultural adaptation}

The translation and cross-cultural adaptation of the IBD-F were performed in five steps: forward translation, synthesis, backward translation, expert committee review, and pretesting. The forward translation was conducted with the intent to retain the meaning of the original scale. The English version of the IBD-F was translated into Brazilian-Portuguese by two independent native Brazilian Portuguese bilingual and bicultural translators, without previous contact with the instrument. One translator had previous knowledge on health sciences. Both translators emphasized the conceptual rather than the literary translation, and produced two independent versions. Whenever a concept had no equivalent description in the Brazilian culture, the appropriate terms were resolved by consensus between the two translators. None of the original items was omitted. In the synthesis step the independent versions were evaluated and compared for the final consensual Portuguese version. The consensual version in Portuguese was back translated into English by two bilingual professional translators neither aware nor informed of the scale concepts being explored. Both translators did not have medical backgrounds to avoid information bias. The two generated English versions were compared with the original text in English by a Brazilian researcher and a university lecturer, who produced a consensual pre-final version for use in the pretesting step.

During the expert committee review, both forward and backward translations were provided to a committee comprised of five bilingual gastroenterologists. Each Committee member independently analyzed the semantic, idiomatic, experiential, and conceptual equivalence of each item in the IBD-F. None of the items were omitted. In this step, an evaluation was made on the readability of the instrument using the legibility scale of Fernandez-Huerta ${ }^{(18)}$. This scale assesses the reading level which is calculated for each 100 -word block of text using the equation: $206.84-(0.60 \times \mathrm{P})-(1.02 \times \mathrm{F})$, where $\mathrm{P}$ is the number of syllables and $\mathrm{F}$ is the number of sentences per 100 words. This first culturally adapted translation was finalized in three months. In the pretesting step the Brazilian-Portuguese version of the IBD-F was administered to 40 patients with IBD.

\section{Phase II - measurement property assessment - Participants}

Participants were recruited from the Gastroenterology Outpatient Clinic of the Federal University of Juiz de Fora University Hospital (HU/UFJF), Dom Bosco Unit. Participants were included if diagnosed with IBD, aged between 18 and 60 years-old, had clinical, endoscopic and histopathological diagnosis of IBD confirmed by the criteria of Ministry of Health for Crohn's disease ${ }^{(20)}$ and ulcerative colitis $^{(21)}$. Patients were excluded if have had any alteration of medication or surgical procedure 30 days prior to participation, were unable to understand the study procedures and assessment instruments or were diagnosed with fibromyalgia or any autoimmune, severe cardiorespiratory, neurological or musculoskeletal diseases. All participants provided written informed consent prior to study participation.

\section{- Assessments}

The participants' demographic and clinical characteristics were collected in the first interview. The Harvey-Bradshaw Index ${ }^{(22)}$ was used as the clinical index of the disease activity in Crohn's disease and the Truelove and Witts ${ }^{(23)}$ was used for ulcerative colitis. For Crohn's disease activity a score of 4 or below was considered as clinical remission while a score of 5 or above considered as the active phase of the disease ${ }^{(24)}$. For ulcerative colitis, clinical remission was defined as $\leq 2$ or 3 stools/day, without the presence of blood and/or pus in the stools and no systemic symptoms; mild activity as up to 4 stools/day, with or without blood, no systemic involvement, and increased inflammatory markers; moderate activity as $>4$ stools per day with minimal systemic symptoms and increased inflammatory markers; and severe activity as $>6$ stools per day with blood and evidence of systemic involvement, such as fever, tachycardia, anemia, and erythrocyte sedimentation above thirty ${ }^{(23)}$. These disease expression indexes were assessed at baseline, 8 and 12-week follow-up assessments.

Fatigue perception was assessed by FACIT-F, a self-reported scale that comprises of 13 items. The total score ranges from 0 to 52, with higher scores indicating lower fatigue. The FACIT-F scale has been used in clinical populations and is valid for use in the Portuguese language ${ }^{(25)}$. Depression and anxiety were assessed by the Hospital Anxiety and Depression Scale (HADS) ${ }^{(26)}$. The HADS has 14 anxiety and depression-related questions, seven in each domain. The responses are rated on a 4-point likertscale, ranging from 0 (no impairment) to 3 (highest impairment). The total score ranges from 0 to 21 and a score of 8 or higher is suggestive of anxiety or depression. In addition, participants were asked to filled out the Brazilian Portuguese-language version of the IBDF scale at three different time-points. The choice of the intervals between testing sessions ( 48 to 72 hours for reproducibility and 8 to 12 weeks for responsiveness) was based on clinical experts' opinion, who indicated considerable clinical changes in 8 to 12-week time in patients with IBD. 


\section{- Data analysis}

The sample size required for Phase II was estimated based on correlation coefficients to explore the association between IBD-F and the FACIT-F. To achieve an alpha value $=0.05$ and power of $90 \%$ for moderate to recommended correlation coefficients $(>0.30)$, a sample size of 85 subjects was needed. The sample size was increased to 123 subjects allowing for possible loss during follow-up. Data distribution was evaluated using the Kolmogorov-Smirnov test. Normal and non-normal distributed data was expressed as mean and standard deviation (SD) or median and range, respectively. The categorical and dichotomous variables were described in frequency and proportion. The measurement properties tested in this study for the Brazilian-Portuguese version of the IBD-F were: internal consistency, reproducibility (ie. reliability and agreement), content validity, internal and external responsiveness and ceiling and floor effects. The receiver operating characteristic (ROC) curve was used to determine the cut-off point with the most appropriate values for specificity and sensitivity in the IBD-F section I, able to discriminate patients with and without fatigue based on the FACITF scores. The data point closest to the curve upper left corner indicated the highest sensitivity and specificity. The Friedman test was used to compare IBD-F score assessed at different timepoints. All analyses were performed using SPSS for Windows version 20.0 (IBM Corporation, Somers, New York, USA).

In addition, each measurement property was tested as recommended ${ }^{(19)}$. The internal consistency (homogeneity) of the IBD-F Brazil was assessed using the Cronbach alpha index and "alpha if item deleted" statistics. The internal consistency analyses the extent to which the items in a given instrument measures different aspects of the same general construct. Estimates greater than 0.70 were considered as adequate ${ }^{(27)}$. The reliability and agreement was used as reproducibility assessment. A two-way intraclass correlation coefficient (ICC) using $95 \%$ confidence intervals was used for reliability. The ICC was considered low if $\leq 0.40$, moderate if $0.40>\mathrm{ICC}<0.75$, substantial if $0.75 \geq \mathrm{ICC}<0.90$, and excellent if ICC $\geq 0.90^{(28)}$. Agreement between the baseline and the assessment following 48 to 72 hours was evaluated by the standard error of the measurement (SEM). Agreement was obtained by the standard deviation of the difference between test and retest divided by $\sqrt{ } 2$. A SEM $\leq 5 \%$ was considered as very good, $5 \%$ to $10 \%$ as good, $11 \%$ to $20 \%$ doubtful and $>20 \%$ as bad agreement ${ }^{(27)}$. The minimum detectable change (MDC) was also evaluated to describe the variability associated with participants' individual scores in the IBD-F and the magnitude of change that a measurement must demonstrate to exceed its anticipated variability. MDC with $95 \%$ confidence was calculated using the formula $\mathrm{MDC}=1.96 / \sqrt{2} \times \mathrm{SEM})^{(28)}$.

The content validity was analysed by calculating the level of association for scores obtained at baseline between the IBD-F Brazil, the FACIT-F and HADS using the Spearman rho correlation coefficients. The correlation coefficients equal to or greater than 0.60 are recommended; correlations ranging from 0.60 to 0.30 are considered as moderate, and lower than 0.30 as weak associations $^{(18)}$. The internal responsiveness of the IBD-F Brazil was measured using the effect-size. Effect size was obtained using the mean differences between the IBD-F Brazil scores at baseline and at 8 to 12 week follow-up assessment divided by the standard deviation obtained at baseline. Effect sizes indicate the questionnaire's sensitivity to measure clinical changes, with a score of $<0.20$ indicate slight, of $\geq 0.20$ to 0.50 as moderate, and of $\geq 0.80$ as a large change ${ }^{(27)}$. The effect-size was estimated for all partici- pants and for the Crohn's disease and ulcerative colitis subgroups separately. To analyse the external responsiveness of the IBD-F Brazil, the correlation between the change score on the HarveyBradshaw Index at 8-12 weeks and the baseline assessment was used for the subgroup of patients with Crohn's disease and the correlation between the change score on the Truelove at 8 to 12 weeks with the change score at 8 to 12 weeks of the IBD-F Brazil for the subgroup of patients with ulcerative colitis. In addition, a receiver-operating-characteristic curve analysis using the FACIT-F was explored. The presence of fatigue was confirmed whenever the IBD-F Brazil cutoff score of 11 points or greater was met. This analysis was based on the area under the curve (AUC), and values $\geq 0.70$ were considered as responsive ${ }^{(18)}$.

The ceiling and floor effects were analysed using the proportion of participants who obtained the maximum and minimum scores of IBD-F Brazil, respectively. Ceiling and floor effects were considered if $15 \%$ or more of the respondents achieved the maximum or minimum scores ${ }^{(27)}$.

\section{RESULTS}

\section{Phase I - translation and cross cultural adaptation of IBD-F}

The IBD-F scale was forward and back translated by a professional and a lay translator, any discrepancies were resolved by the expert committee. The expert committee decided to replace item 11 in the Section II, "I had difficulty continuing with my hobbies/ interests because of fatigue" to "I had difficulty continuing my leisure activities because of fatigue" and item 12 in the Section II, "My emotional relationship with my partner was affected by fatigue" to "My affective relationship with my partner was affected by fatigue". These changes were made based on colloquial Brazilian-Portuguese while keeping the same meaning of the sentence. All questions and options answered by 40 patients assessed in Phase I were understandable and applicable to clinical context. The demographic and clinical characteristics of participants included in Phase I and II are summarized in TABLE 1. The Brazilian-Portuguese versions of the IBD-F did not require additional changes and the number of items and scoring process from the original English versions were maintained, with higher scores indicating higher level of fatigue. The readability in the legibility scale of Fernandez-Huerta was 83.6, which indicate good text readability, appropriate to the target population. The full cross-cultural adaptation process used is described in FIGURE 1 and the version of the cross-culturally adapted IBD-F Brazil scale is available in FIGURE 2.

\section{Phase II of the study - measurement properties testing}

A total of 123 participants were assessed on Phase II. The dropout rate was four percent at the third assessment due to loss of patient contact, 118 participants completed the survey (81 patients with Crohn's disease and 37 with ulcerative colitis). At baseline, participants were asked to complete the IBD-F, the FACIT-F and the HADS instruments. The IBD-F Brazil was also applied following 48 to 72 hours and 8 to 12 weeks following initial assessment. Disease activity was assessed at baseline and at 8 to 12 weeks.

The results of the IBD-F Brazil measurement properties assessed are reported in TABLE 2. The internal consistency of the IBD-F Brazil was adequate for both Sections of the questionnaire. The Cronbach alpha indexes were 0.95 and 0.98 for Sections I and II, respectively. None of the scale items increased reliability when 
TABLE 1 . Demographic and clinical characteristics of the participants.

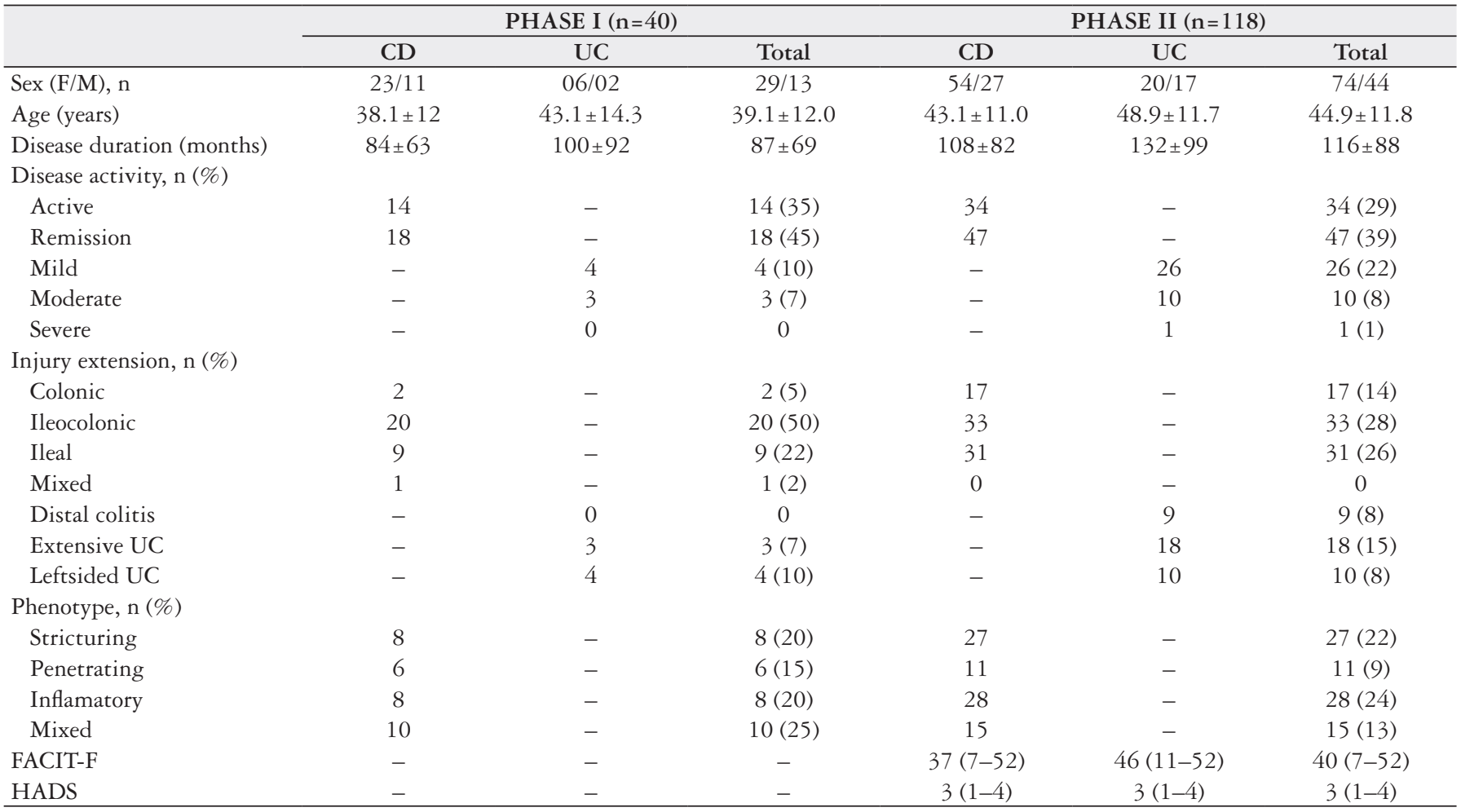

Data presented in n (\%), mean \pm SD and median (range). CD: Crohn's disease; F: female; FACIT-F: Functional Assessment of Chronic Illness Therapy-Fatigue; HADS: Hospital Anxiety and Depression Scale; M: male; UC: ulcerative colitis.

APÊNDICE C - Inflammatory Bowel Disease-Fatigue Patient Self-Assessment Scale (IBD-F): IBD-F Brasil

Data:

Hora:

\section{SEÇÃO I - Escala de avaliação da fadiga}

Esta seção do questionário identificará o que é fadiga, sua gravidade, frequência e duração.

Às vezes, pessoas portadoras de doença inflamatória intestinal se sentem fatigadas. O termo "fadiga" é usado em todo o questionário. A fadiga foi definida como uma sensação de cansaço contínuo, com períodos de repentina e intensa falta de energia ou sensação de exaustão que não é aliviada após o repouso ou o sono.

Por favor, assinale apenas UM número para $\quad$ Avalie de 0 a 4 , em que cada pergunta

$0=$ sem fadiga

Fadiga intensa $=4$

\begin{tabular}{|c|c|c|c|c|c|}
\hline 1. Qual é o seu nível de fadiga AGORA? & 0 & 1 & 2 & 3 & 4 \\
\hline $\begin{array}{l}\text { 2. Qual foi o seu MAIOR nível de fadiga nas últimas duas } \\
\text { semanas? }\end{array}$ & 0 & 1 & 2 & 3 & 4 \\
\hline $\begin{array}{l}\text { 3. Qual foi o seu MENOR nível de fadiga nas últimas } \\
\text { duas semanas? }\end{array}$ & 0 & 1 & 2 & 3 & 4 \\
\hline $\begin{array}{l}\text { 4. Qual foi o seu nível MÉDIO de fadiga nas últimas duas } \\
\text { semanas? }\end{array}$ & 0 & 1 & 2 & 3 & 4 \\
\hline $\begin{array}{l}\text { 5. Durante o tempo em que estava acordado, com que } \\
\text { frequência você se sentiu fatigado nas últimas duas } \\
\text { semanas? }\end{array}$ & $\begin{array}{c}\mathbf{0} \\
\text { Nenhuma } \\
\text { das vezes }\end{array}$ & $\begin{array}{c}1 \\
\text { Algumas } \\
\text { vezes }\end{array}$ & $\begin{array}{l}2 \\
\text { Muitas } \\
\text { vezes }\end{array}$ & $\begin{array}{c}3 \\
\text { A maior } \\
\text { parte } \\
\text { do } \\
\text { tempo }\end{array}$ & $\begin{array}{l}4 \\
\text { O } \\
\text { tempo } \\
\text { todo }\end{array}$ \\
\hline
\end{tabular}

FIGURE 1. IBD-F Brazil. 


\section{SEÇÃO II - Escala de impacto da fadiga nas atividades diárias na DII}

Esta seção avalia o impacto da fadiga percebido em suas atividades diárias nas últimas duas semanas.

Por favor, responda a todas as perguntas. As possíveis respostas às perguntas são:

Nenhuma das vezes - 0; Algumas vezes - 1; Muitas vezes - 2; A maior parte do tempo - 3; O tempo todo - 4;

Se uma atividade específica não se aplicar a você, selecione Não se Aplica (N/A). Por exemplo, se você não dirigir, selecione N/A.

\begin{tabular}{|c|c|c|c|c|c|c|}
\hline $\begin{array}{l}\text { Por favor, concentre-se nas duas últimas semanas } \\
\text { e assinale apenas UMA resposta para cada } \\
\text { pergunta }\end{array}$ & $\begin{array}{l}\text { Nenhuma } \\
\text { das vezes }\end{array}$ & $\begin{array}{c}\text { Algumas } \\
\text { vezes }\end{array}$ & $\begin{array}{l}\text { Muitas } \\
\text { vezes }\end{array}$ & $\begin{array}{l}\text { A maior } \\
\text { parte do } \\
\text { tempo }\end{array}$ & $\begin{array}{l}\text { O tempo } \\
\text { todo }\end{array}$ & $\begin{array}{l}\text { Não se } \\
\text { aplica }\end{array}$ \\
\hline
\end{tabular}

\begin{tabular}{|c|c|c|c|c|c|c|}
\hline 1. Precisei cochilar durante o dia por causa da fadiga & 0 & 1 & 2 & 3 & 4 & \\
\hline 2. A fadiga me impediu de comparecer a eventos sociais & s 0 & 1 & 2 & 3 & 4 & \\
\hline $\begin{array}{l}\text { 3. Não fui capaz de ir ao trabalho ou à faculdade por } \\
\text { causa da fadiga }\end{array}$ & 0 & 1 & 2 & 3 & 4 & N/A \\
\hline $\begin{array}{l}\text { 4. Meu desempenho no trabalho ou nos estudos foi } \\
\text { afetado pela fadiga }\end{array}$ & 0 & 1 & 2 & 3 & 4 & N/A \\
\hline 5. Tive problemas de concentração por causa da fadiga & 0 & 1 & 2 & 3 & 4 & \\
\hline 6. Tive dificuldade em me motivar por causa da fadiga & 0 & 1 & 2 & 3 & 4 & \\
\hline $\begin{array}{l}\text { 7. Não consegui tomar banho e me vestir por causa da } \\
\text { fadiga }\end{array}$ & 0 & 1 & 2 & 3 & 4 & \\
\hline 8. Tive dificuldade em caminhar por causa da fadiga & 0 & 1 & 2 & 3 & 4 & \\
\hline $\begin{array}{l}\text { 9. Não consegui dirigir o tanto que precisava por causa } \\
\text { da fadiga }\end{array}$ & 0 & 1 & 2 & 3 & 4 & N/A \\
\hline $\begin{array}{l}\text { 10. Não fui capaz de fazer tantos exercícios físicos } \\
\text { como eu gostaria por causa da fadiga }\end{array}$ & 0 & 1 & 2 & 3 & 4 & \\
\hline $\begin{array}{l}\text { Por favor, concentre-se nas duas últimas semanas } \\
\text { e assinale apenas UMA resposta para cada } \\
\text { pergunta }\end{array}$ & $\begin{array}{l}\text { Nenhuma } \\
\text { das vezes }\end{array}$ & $\begin{array}{l}\text { Algumas } \\
\text { vezes }\end{array}$ & $\begin{array}{c}\text { Muitas } \\
\text { vezes }\end{array}$ & $\begin{array}{l}\text { A maior } \\
\text { parte do } \\
\text { tempo }\end{array}$ & $\begin{array}{l}\text { O tempo } \\
\text { todo }\end{array}$ & $\begin{array}{l}\text { Não se } \\
\text { aplica }\end{array}$ \\
\hline $\begin{array}{l}\text { 11. Tive dificuldade em continuar com minhas atividades } \\
\text { de lazer por causa da fadiga }\end{array}$ & 0 & 1 & 2 & 3 & 4 & \\
\hline $\begin{array}{l}\text { 12. Meu relacionamento afetivo com meu/minha } \\
\text { parceiro(a) foi afetado pela fadiga }\end{array}$ & 0 & 1 & 2 & 3 & 4 & N/A \\
\hline $\begin{array}{l}\text { 13. Meu relacionamento sexual com meu/minha } \\
\text { parceiro(a) foi afetado pela fadiga }\end{array}$ & 0 & 1 & 2 & 3 & 4 & N/A \\
\hline $\begin{array}{l}\text { 14. Meu relacionamento com meus filhos foi afetado pela } \\
\text { fadiga }\end{array}$ & 0 & 1 & 2 & 3 & 4 & N/A \\
\hline 15. Fiquei de mau humor por causa da fadiga & 0 & 1 & 2 & 3 & 4 & \\
\hline 16. Senti-me isolado por causa da fadiga & 0 & 1 & 2 & 3 & 4 & \\
\hline 17. Minha memória foi afetada por causa da fadiga & 0 & 1 & 2 & 3 & 4 & \\
\hline 18. Cometi erros por causa da fadiga & 0 & 1 & 2 & 3 & 4 & \\
\hline 19. A fadiga me deixou irritado & 0 & 1 & 2 & 3 & 4 & \\
\hline 20. A fadiga me deixou frustrado & 0 & 1 & 2 & 3 & 4 & \\
\hline $\begin{array}{l}\text { 21. Ao me expressar, troco as palavras por causa da } \\
\text { fadiga }\end{array}$ & 0 & 1 & 2 & 3 & 4 & \\
\hline 22. A fadiga me impediu de aproveitar a vida & 0 & 1 & 2 & 3 & 4 & \\
\hline 23. A fadiga me impediu de ter uma vida plena & 0 & 1 & 2 & 3 & 4 & \\
\hline 24. Minha autoestima foi afetada pela fadiga & 0 & 1 & 2 & 3 & 4 & \\
\hline 25. A fadiga afetou minha confiança & 0 & 1 & 2 & 3 & 4 & \\
\hline
\end{tabular}




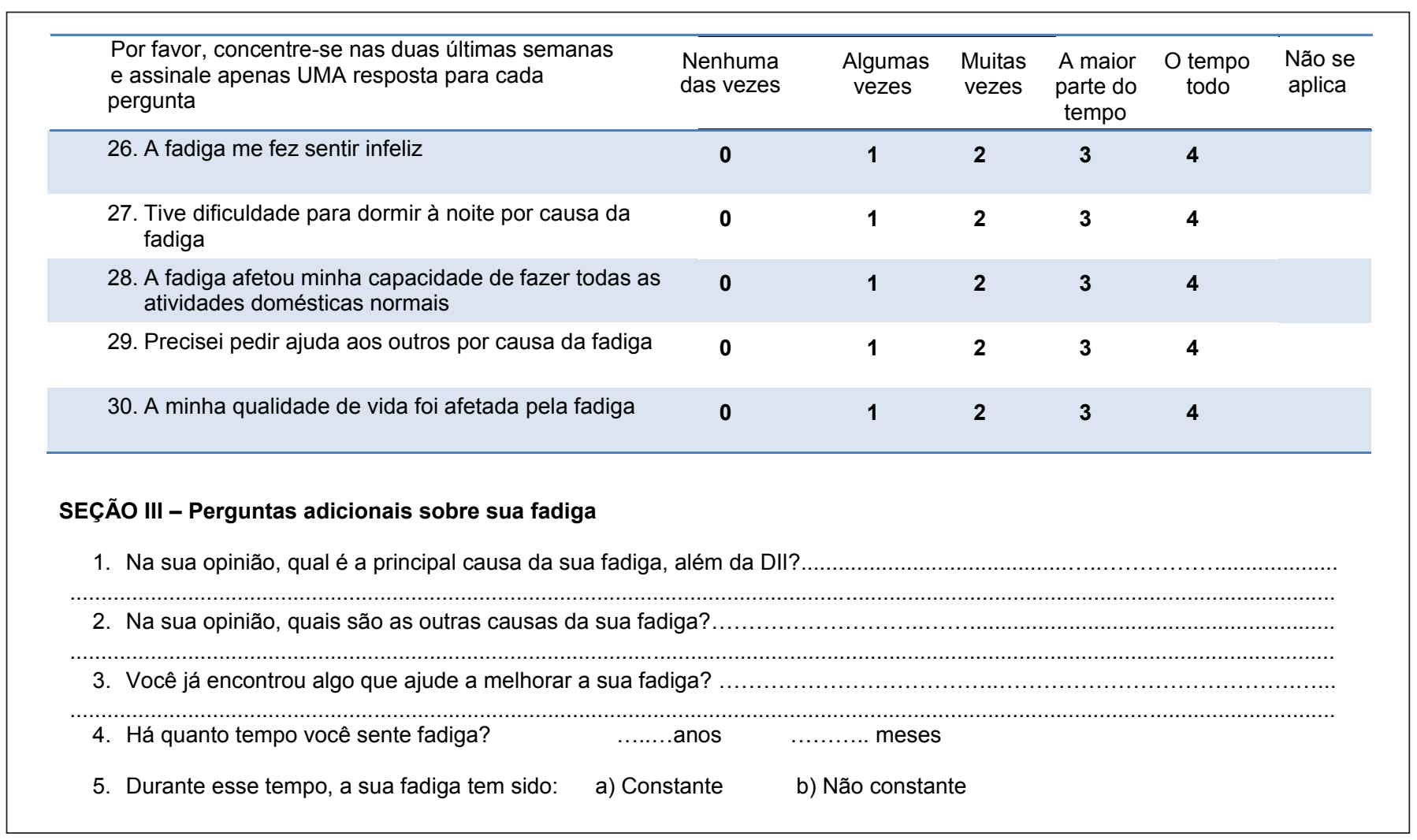

FIGURE 1: end.

\begin{tabular}{|c|c|c|c|c|}
\hline \multicolumn{5}{|c|}{ APÊNDICE D - Processo de Adaptação Transcultural } \\
\hline Versão Original & Traduções & $\begin{array}{c}\text { Consenso da Versão } \\
\text { Port.-Brasileira }\end{array}$ & Retrotraduções & $\begin{array}{l}\text { Versão Final } \\
\text { Port.Brasileira }\end{array}$ \\
\hline $\begin{array}{l}\text { SESSÃO I: } \\
\text { 1. What is your fatigue level right } \\
\text { NOW }\end{array}$ & $\begin{array}{l}\text { T1: Qual é o seu nível de } \\
\text { fadiga AGORA } \\
\text { T2: 1. Qual é o seu nível } \\
\text { de fadiga AGORA }\end{array}$ & $\begin{array}{l}\text { Qual é o seu nível de } \\
\text { fadiga AGORA? }\end{array}$ & $\begin{array}{l}\text { R1: What is your level of fatigue } \\
\text { AT THIS MOMENT? } \\
\text { R2: What is your level of fatigue } \\
\text { NOW? }\end{array}$ & $\begin{array}{l}\text { Qual é o seu nível de } \\
\text { fadiga AGORA? }\end{array}$ \\
\hline $\begin{array}{l}\text { 2. What was your HIGHEST } \\
\text { fatigue level in the past two } \\
\text { weeks }\end{array}$ & $\begin{array}{l}\text { T1: Qual foi o seu MAIOR } \\
\text { nível de fadiga nas últimas } \\
\text { duas semanas } \\
\text { T2: Qual foi o seu MAIOR } \\
\text { nível de fadiga nas últimas } \\
\text { duas semanas }\end{array}$ & $\begin{array}{l}\text { Qual foi o seu MAIOR } \\
\text { nível de fadiga nas } \\
\text { últimas duas semanas? }\end{array}$ & $\begin{array}{l}\text { R1: How would you rate your } \\
\text { HIGHEST level of fatigue } \\
\text { during the last two weeks? } \\
\text { R2: What was your HIGHEST } \\
\text { level of fatigue in the last two } \\
\text { weeks? }\end{array}$ & $\begin{array}{l}\text { Qual foi o seu MAIOR } \\
\text { nível de fadiga nas } \\
\text { últimas duas semanas? }\end{array}$ \\
\hline $\begin{array}{l}\text { 3. What was your LOWEST } \\
\text { fatigue level in the past two } \\
\text { weeks }\end{array}$ & $\begin{array}{l}\text { T1: Qual foi o seu MENOR } \\
\text { nível de fadiga nas últimas } \\
\text { duas semanas } \\
\text { T2: Qual foi o seu MENOR } \\
\text { nível de fadiga nas ultimas } \\
\text { duas semanas }\end{array}$ & $\begin{array}{l}\text { Qual foi o seu MENOR } \\
\text { nível de fadiga nas } \\
\text { últimas duas semanas? }\end{array}$ & $\begin{array}{l}\text { R1: How would you rate your } \\
\text { LOWEST level of fatigue during } \\
\text { the last two weeks? } \\
\text { R2: What was the LOWEST } \\
\text { level of fatigue in the last two } \\
\text { weeks? }\end{array}$ & $\begin{array}{l}\text { Qual foi o seu MENOR } \\
\text { nível de fadiga nas } \\
\text { últimas duas semanas? }\end{array}$ \\
\hline
\end{tabular}

FIGURE 2. The IBD-F original version, itens and translations, the Brazilian-Portuguese version following consensus, back-translations and the IBD-F Brazil final version. 
4. What was your AVERAGE fatigue level in the past two weeks
T1: Qual foi o seu nível MÉDIO de fadiga nas últimas duas semanas

T2: Qual foi o seu nível MÉDIO de fadiga nas últimas duas semanas
Qual foi o seu nível MÉDIO de fadiga nas últimas duas semanas?
R1: What was your AVERAGE level of fatigue during the last two weeks?

R2: What was your AVERAGE level of fatigue in the last two weeks?
Qual foi o seu nível MÉDIO de fadiga nas últimas duas semanas?
T1: Durante o tempo em que estava acordado, com que frequência você se sentiu fatigado nas últimas duas semanas

5. How much of your waking time have you felt fatigued in the past two weeks

T2: Quanto de seu tempo acordado você se sentiu fatigado nas últimas duas semanas
R1: During the time that you were awake, how many times did you feel fatigued in the last two weeks?
Durante o tempo em que estava acordado, com que frequência você se sentiu fatigado nas últimas duas semanas?

R2: During the time that you were awake, how often did you feel fatigued in the last two weeks?
Durante o tempo em que estava acordado, com que frequência você se sentiu fatigado nas últimas duas semanas?

\section{SESSÃO II:}

1. I had to nap during the day because of fatigue

\section{T1: Precisei cochilar} durante o dia por causa da fadiga

T2: Eu tive que cochilar durante o dia por causa da fadiga
R1: I needed to take a nap during the day because of

Precisei cochilar durante fatigue o dia por causa da fadiga

R2: I needed to take a nap during the day because of fatigue
T1: A fadiga me impediu 2. Fatigue stopped me from going de comparecer a eventos out to social events
A fadiga me impediu de comparecer a eventos sociais
R1: Fatigue inhibited me from going to social events

R2: Fatigue prevented me from going to social events
Precisei cochilar durante o dia por causa da fadiga
T2: A fadiga me impediu de sair para eventos sociais faculdade por causa da fadiga

T2: Eu não era capaz de ir para o trabalho ou faculdade por causa da fadiga

T1: Meu desempenho no trabalho ou nos estudos foi afetado pela fadiga

4. My performance at work or education was affected by fatigue

T2: Meu desempenho no trabalho ou nos estudos foi afetado pela fadiga
T1: Faltei ao trabalho ou à
Não fui capaz de ir ao trabalho ou à faculdade por causa da fadiga
R1: I was unable to go to work or school due to fatigue

Não fui capaz de ir ao trabalho ou à faculdade por causa da fadiga
R2: I was unable to go to work or to university because of fatigue

A fadiga me impediu de comparecer a eventos

Meu desempenho no
Meu desempenho no trabalho ou nos estudos foi afetado pela fadiga
T1: Tive problemas de concentração por causa da fadiga

5. I had problems concentrating because of fatigue
T2: Tive problemas de concentração por causa da fadiga
Tive problemas de concentração por causa da fadiga trabalho ou nos estudos foi afetado pela fadiga

R1: My performance at work or school was affected by fatigue

R2: My productivity at work or in my studies was affected by fatigue

\section{R1: I had problems} concentrating because of fatigue

R2: I had problems concentrating because of fatigue
Tive problemas de concentração por causa da fadiga 


\begin{tabular}{|c|c|c|c|c|}
\hline $\begin{array}{l}\text { 6. I had difficulty motivating } \\
\text { myself because of fatigue }\end{array}$ & $\begin{array}{l}\text { T1: Tive dificuldade em me } \\
\text { motivar por causa da } \\
\text { fadiga } \\
\text { T2: Tive dificuldade em me } \\
\text { motivar por causa da } \\
\text { fadiga }\end{array}$ & $\begin{array}{l}\text { Tive dificuldade em me } \\
\text { motivar por causa da } \\
\text { fadiga }\end{array}$ & $\begin{array}{l}\text { R1: I had difficulty in motivating } \\
\text { myself due to fatigue } \\
\text { R2: I had difficulty motivating } \\
\text { myself because of fatigue }\end{array}$ & $\begin{array}{l}\text { Tive dificuldade em me } \\
\text { motivar por causa da } \\
\text { fadiga }\end{array}$ \\
\hline $\begin{array}{l}\text { 7. I could not wash and dress } \\
\text { myself because of fatigue }\end{array}$ & $\begin{array}{l}\text { T1: Não consegui tomar } \\
\text { banho nem me vestir por } \\
\text { causa da fadiga } \\
\text { T2: Não consegui tomar } \\
\text { banho e vestir-me por } \\
\text { causa da fadiga }\end{array}$ & $\begin{array}{l}\text { Não consegui tomar } \\
\text { banho e me vestir por } \\
\text { causa da fadiga }\end{array}$ & $\begin{array}{l}\text { R1: I was unable to bathe or } \\
\text { dress myself due to fatigue } \\
\text { R2: I was unable to take a } \\
\text { shower and dress myself } \\
\text { because of fatigue }\end{array}$ & $\begin{array}{l}\text { Não consegui tomar } \\
\text { banho e me vestir por } \\
\text { causa da fadiga }\end{array}$ \\
\hline $\begin{array}{l}8 . \text { I had difficulty with walking } \\
\text { because of fatigue }\end{array}$ & $\begin{array}{l}\text { T1: Tive dificuldade em } \\
\text { caminhar por causa da } \\
\text { fadiga } \\
\text { T2: Tive dificuldade em } \\
\text { caminhar por causa da } \\
\text { fadiga }\end{array}$ & $\begin{array}{l}\text { Tive dificuldade em } \\
\text { caminhar por causa da } \\
\text { fadiga }\end{array}$ & $\begin{array}{l}\text { R1: I had difficulty walking due } \\
\text { to fatigue } \\
\text { R2: I had difficulty walking } \\
\text { because of fatigue }\end{array}$ & $\begin{array}{l}\text { Tive dificuldade em } \\
\text { caminhar por causa da } \\
\text { fadiga }\end{array}$ \\
\hline $\begin{array}{l}\text { 9. I was unable to drive as much } \\
\text { as I need to because of fatigue }\end{array}$ & $\begin{array}{l}\text { T1: Não consegui dirigir o } \\
\text { tanto que precisava por } \\
\text { causa da fadiga } \\
\text { T2: Não fui capaz de dirigir } \\
\text { tanto quanto eu preciso } \\
\text { por causa da fadiga }\end{array}$ & $\begin{array}{l}\text { Não consegui dirigir o } \\
\text { tanto que precisava por } \\
\text { causa da fadiga }\end{array}$ & $\begin{array}{l}\text { R1: I was unable to drive as } \\
\text { much as I needed to because } \\
\text { of fatigue } \\
\text { R2: I was unable to drive as } \\
\text { much as I needed to because } \\
\text { of fatigue }\end{array}$ & $\begin{array}{l}\text { Não consegui dirigir o } \\
\text { tanto que precisava por } \\
\text { causa da fadiga }\end{array}$ \\
\hline $\begin{array}{l}\text { 10. I was not able to do as much } \\
\text { physical exercise as I wanted to } \\
\text { because of fatigue }\end{array}$ & $\begin{array}{l}\text { T1: Não consegui praticar } \\
\text { exercícios físicos o tanto } \\
\text { que queria por causa da } \\
\text { fadiga } \\
\text { T2: Não fui capaz de fazer } \\
\text { tanto exercício físico como } \\
\text { eu queria por causa da } \\
\text { fadiga }\end{array}$ & $\begin{array}{l}\text { Não fui capaz de fazer } \\
\text { tantos exercícios físicos } \\
\text { como eu gostaria por } \\
\text { causa da fadiga }\end{array}$ & $\begin{array}{l}\text { R1: I wasn't able to do as much } \\
\text { physical exercise as I would } \\
\text { have liked to due to fatigue } \\
\text { R2: I was unable to exercise as } \\
\text { much as I would have liked } \\
\text { because of fatigue }\end{array}$ & $\begin{array}{l}\text { Não fui capaz de fazer } \\
\text { tantos exercícios físicos } \\
\text { como eu gostaria por } \\
\text { causa da fadiga }\end{array}$ \\
\hline $\begin{array}{l}\text { 11. I had difficulty continuing with } \\
\text { my hobbies/interests because of } \\
\text { fatigue }\end{array}$ & $\begin{array}{l}\text { T1: Tive dificuldade em } \\
\text { continuar com meus } \\
\text { hobbies/interesses por } \\
\text { causa da fadiga } \\
\text { T2: Tive dificuldade em } \\
\text { continuar com meus } \\
\text { hobbies/interesses por } \\
\text { causa da fadiga }\end{array}$ & $\begin{array}{l}\text { Tive dificuldade em } \\
\text { continuar com meus } \\
\text { hobbies/interesses por } \\
\text { causa da fadiga }\end{array}$ & $\begin{array}{l}\text { R1: I had problems performing } \\
\text { my hobbies/activities due to } \\
\text { fatigue } \\
\text { R2: I had difficulty participating } \\
\text { in my hobbies/interests } \\
\text { because of fatigue }\end{array}$ & $\begin{array}{l}\text { Tive dificuldade em } \\
\text { continuar com minhas } \\
\text { atividades de lazer por } \\
\text { causa da fadiga }\end{array}$ \\
\hline $\begin{array}{l}\text { 12. My emotional relationship } \\
\text { with my partner was affected by } \\
\text { fatigue }\end{array}$ & $\begin{array}{l}\text { T1: Meu relacionamento } \\
\text { emocional com meu/minha } \\
\text { parceiro(a) foi afetado pela } \\
\text { fadiga } \\
\text { T2: Minha relação } \\
\text { emocional com meu } \\
\text { parceiro foi afetada pela } \\
\text { fadiga }\end{array}$ & $\begin{array}{l}\text { Meu relacionamento } \\
\text { emocional com } \\
\text { meu/minha parceiro(a) } \\
\text { foi afetado pela fadiga }\end{array}$ & $\begin{array}{l}\text { R1: My emotional relationship } \\
\text { with my partner was affected by } \\
\text { fatigue } \\
\text { R2: My emotional relationship } \\
\text { with my partner was affected by } \\
\text { fatigue }\end{array}$ & $\begin{array}{l}\text { Meu relacionamento } \\
\text { afetivo com meu/minha } \\
\text { parceiro(a) foi afetado } \\
\text { pela fadiga }\end{array}$ \\
\hline
\end{tabular}




\begin{tabular}{|c|c|c|c|c|}
\hline \multirow[t]{2}{*}{$\begin{array}{l}\text { 13. My sexual relationship with } \\
\text { my partner was affected by } \\
\text { fatigue }\end{array}$} & $\begin{array}{l}\text { T1: Meu relacionamento } \\
\text { sexual com meu/minha } \\
\text { parceiro(a) foi afetado pela } \\
\text { fadiga }\end{array}$ & \multirow[t]{2}{*}{$\begin{array}{l}\text { Meu relacionamento } \\
\text { sexual com meu/minha } \\
\text { parceiro(a) foi afetado } \\
\text { pela fadiga }\end{array}$} & $\begin{array}{l}\text { R1: My sexual relationship with } \\
\text { my partner was affected by } \\
\text { fatigue }\end{array}$ & \multirow[t]{2}{*}{$\begin{array}{l}\text { Meu relacionamento } \\
\text { sexual com meu/minha } \\
\text { parceiro(a) foi afetado } \\
\text { pela fadiga }\end{array}$} \\
\hline & $\begin{array}{l}\text { T2: Minha relação sexual } \\
\text { com meu parceiro foi } \\
\text { afetada pela fadiga }\end{array}$ & & $\begin{array}{l}\text { R2: My sexual relationship with } \\
\text { my partner was affected by } \\
\text { fatigue }\end{array}$ & \\
\hline \multirow[t]{2}{*}{$\begin{array}{l}\text { 14. My relationship with my } \\
\text { children was affected by fatigue }\end{array}$} & $\begin{array}{l}\text { T1: Meu relacionamento } \\
\text { com meus filhos foi } \\
\text { afetado pela fadiga }\end{array}$ & $\begin{array}{l}\text { Meu relacionamento com } \\
\text { meus filhos foi afetada } \\
\text { pela fadiga }\end{array}$ & $\begin{array}{l}\text { R1: My relationship with my } \\
\text { children was affected by fatigue }\end{array}$ & $\begin{array}{l}\text { Meu relacionamento } \\
\text { com meus filhos foi } \\
\text { afetada pela fadiga }\end{array}$ \\
\hline & $\begin{array}{l}\text { T2: Minha relação com } \\
\text { meus filhos foi afetada } \\
\text { pela fadiga }\end{array}$ & & $\begin{array}{l}\text { R2: My relationship with my } \\
\text { children was affected by fatigue }\end{array}$ & \\
\hline \multirow[t]{2}{*}{$\begin{array}{l}\text { 15. I was low in mood because of } \\
\text { fatigue }\end{array}$} & $\begin{array}{l}\text { T1: Fiquei de mau humor } \\
\text { por causa da fadiga }\end{array}$ & $\begin{array}{l}\text { Fiquei de mau humor por } \\
\text { causa da fadiga }\end{array}$ & $\begin{array}{l}\mathrm{R} 1 \text { : I was in a bad mood due to } \\
\text { fatigue }\end{array}$ & $\begin{array}{l}\text { Fiquei de mau humor } \\
\text { por causa da fadiga }\end{array}$ \\
\hline & $\begin{array}{l}\text { T2: Estava com mau } \\
\text { humor por causa da fadiga }\end{array}$ & & $\begin{array}{l}\mathrm{R} 2 \text { : I was in a bad mood } \\
\text { because of fatigue }\end{array}$ & \\
\hline \multirow[t]{2}{*}{$\begin{array}{l}\text { 16. I felt isolated because of } \\
\text { fatigue }\end{array}$} & $\begin{array}{l}\text { T1: Senti-me isolado por } \\
\text { causa da fadiga }\end{array}$ & $\begin{array}{l}\text { Senti-me isolado por } \\
\text { causa da fadiga }\end{array}$ & $\begin{array}{l}\mathrm{R} 1 \text { : I felt isolated because of } \\
\text { fatigue }\end{array}$ & $\begin{array}{l}\text { Senti-me isolado por } \\
\text { causa da fadiga }\end{array}$ \\
\hline & $\begin{array}{l}\text { T2: Senti-me isolado por } \\
\text { causa da fadiga }\end{array}$ & & $\begin{array}{l}\text { R2: I felt isolated because of } \\
\text { fatigue }\end{array}$ & \\
\hline \multirow[t]{2}{*}{$\begin{array}{l}\text { 17. My memory was affected } \\
\text { because of fatigue }\end{array}$} & $\begin{array}{l}\text { T1: Minha memória foi } \\
\text { afetada por causa da } \\
\text { fadiga }\end{array}$ & $\begin{array}{l}\text { Minha memória foi } \\
\text { afetada por causa da } \\
\text { fadiga }\end{array}$ & $\begin{array}{l}\text { R1: My memory was affected } \\
\text { because of fatigue }\end{array}$ & $\begin{array}{l}\text { Minha memória foi } \\
\text { afetada por causa da } \\
\text { fadiga }\end{array}$ \\
\hline & $\begin{array}{l}\text { T2: Minha memória foi } \\
\text { afetada por causa da } \\
\text { fadiga }\end{array}$ & & $\begin{array}{l}\text { R2: My memory was affected } \\
\text { because of fatigue }\end{array}$ & \\
\hline \multirow[t]{2}{*}{$\begin{array}{l}\text { 18. I made mistakes because of } \\
\text { fatigue }\end{array}$} & $\begin{array}{l}\text { T1: Cometi erros por } \\
\text { causa da fadiga }\end{array}$ & $\begin{array}{l}\text { Cometi erros por causa } \\
\text { da fadiga }\end{array}$ & $\begin{array}{l}\text { R1: I made mistakes due to } \\
\text { fatigue }\end{array}$ & $\begin{array}{l}\text { Cometi erros por causa } \\
\text { da fadiga }\end{array}$ \\
\hline & $\begin{array}{l}\text { T2: Cometi erros por } \\
\text { causa da fadiga }\end{array}$ & & $\begin{array}{l}\text { R2: I made mistakes because } \\
\text { of fatigue }\end{array}$ & \\
\hline \multirow[t]{2}{*}{ 19. Fatigue made me irritable } & $\begin{array}{l}\text { T1: A fadiga me deixou } \\
\text { irritado }\end{array}$ & $\begin{array}{l}\text { A fadiga me deixou } \\
\text { irritado }\end{array}$ & $\begin{array}{l}\text { R1: Fatigue left me feeling } \\
\text { irritated }\end{array}$ & $\begin{array}{l}\text { A fadiga me deixou } \\
\text { irritado }\end{array}$ \\
\hline & $\begin{array}{l}\text { T2: A fadiga me deixou } \\
\text { irritado }\end{array}$ & & $\begin{array}{l}\text { R2: Fatigue made me feel } \\
\text { irritated }\end{array}$ & \\
\hline \multirow[t]{2}{*}{ 20. Fatigue made me frustrated } & $\begin{array}{l}\text { T1: A fadiga me deixou } \\
\text { frustrado }\end{array}$ & $\begin{array}{l}\text { A fadiga me deixou } \\
\text { frustrado }\end{array}$ & $\begin{array}{l}\text { R1: Fatigue left me feeling } \\
\text { frustrated }\end{array}$ & $\begin{array}{l}\text { A fadiga me deixou } \\
\text { frustrado }\end{array}$ \\
\hline & $\begin{array}{l}\text { T2: A fadiga me deixou } \\
\text { frustrado }\end{array}$ & & $\begin{array}{l}\text { R2: Fatigue made me feel } \\
\text { frustrated }\end{array}$ & \\
\hline \multirow[t]{2}{*}{$\begin{array}{l}\text { 21. I got words mixed up because } \\
\text { of fatigue }\end{array}$} & $\begin{array}{l}\text { T1: Misturei as palavras } \\
\text { por causa da fadiga }\end{array}$ & $\begin{array}{l}\text { Ao me expressar, troco } \\
\text { as palavras por causa da } \\
\text { fadiga }\end{array}$ & $\begin{array}{l}\text { R1: I found myself mixing up } \\
\text { words due to fatigue }\end{array}$ & $\begin{array}{l}\text { Ao me expressar, troco } \\
\text { as palavras por causa } \\
\text { da fadiga }\end{array}$ \\
\hline & $\begin{array}{l}\text { T2: Ao me expressar, } \\
\text { troco as palavras por } \\
\text { causa da fadiga }\end{array}$ & & $\begin{array}{l}\text { R2: When I express myself, my } \\
\text { phrasing is awkward because } \\
\text { of fatigue }\end{array}$ & \\
\hline \multirow[t]{2}{*}{$\begin{array}{l}\text { 22. Fatigue stopped me from } \\
\text { enjoying life }\end{array}$} & $\begin{array}{l}\text { T1: A fadiga me impediu } \\
\text { de desfrutar a vida }\end{array}$ & $\begin{array}{l}\text { A fadiga me impediu de } \\
\text { aproveitar a vida }\end{array}$ & $\begin{array}{l}\text { R1: Fatigue kept me from } \\
\text { enjoying life }\end{array}$ & $\begin{array}{l}\text { A fadiga me impediu de } \\
\text { aproveitar a vida }\end{array}$ \\
\hline & $\begin{array}{l}\text { T2: A fadiga me impediu } \\
\text { de desfrutar da vida }\end{array}$ & & $\begin{array}{l}\text { R2: Fatigue prevented me from } \\
\text { enjoying life }\end{array}$ & \\
\hline \multirow[t]{2}{*}{$\begin{array}{l}\text { 23. Fatigue stopped me from } \\
\text { having a fulfilling life }\end{array}$} & $\begin{array}{l}\text { T1: A fadiga me impediu } \\
\text { de viver a vida plenamente }\end{array}$ & $\begin{array}{l}\text { A fadiga me impediu de } \\
\text { ter uma vida plena }\end{array}$ & $\begin{array}{l}\text { R1: Fatigue kept me from } \\
\text { having a full life }\end{array}$ & $\begin{array}{l}\text { A fadiga me impediu de } \\
\text { ter uma vida plena }\end{array}$ \\
\hline & $\begin{array}{l}\text { T2: A fadiga me impediu } \\
\text { de ter uma vida plena }\end{array}$ & & $\begin{array}{l}\text { R2: Fatigue prevented me from } \\
\text { having a full life }\end{array}$ & \\
\hline
\end{tabular}




\begin{tabular}{|c|c|c|c|c|}
\hline $\begin{array}{l}\text { 24. My self-esteem was affected } \\
\text { by fatigue }\end{array}$ & $\begin{array}{l}\text { T1: Minha autoestima foi } \\
\text { afetada pela fadiga } \\
\text { T2: Minha auto-estima foi } \\
\text { afetada pela fadiga }\end{array}$ & $\begin{array}{l}\text { Minha autoestima foi } \\
\text { afetada pela fadiga }\end{array}$ & $\begin{array}{l}\text { R1: My self-esteem was } \\
\text { affected by my fatigue } \\
\text { R2: My self-esteem was } \\
\text { affected by fatigue }\end{array}$ & $\begin{array}{l}\text { Minha autoestima foi } \\
\text { afetada pela fadiga }\end{array}$ \\
\hline $\begin{array}{l}\text { 25. Fatigue affected my } \\
\text { confidence }\end{array}$ & $\begin{array}{l}\text { T1: A fadiga afetou minha } \\
\text { confiança } \\
\text { T2: A fadiga afetou minha } \\
\text { confiança }\end{array}$ & $\begin{array}{l}\text { A fadiga afetou minha } \\
\text { confiança }\end{array}$ & $\begin{array}{l}\text { R1: Fatigue affected my } \\
\text { confidence } \\
\text { R2: Fatigue affected my } \\
\text { confidence }\end{array}$ & $\begin{array}{l}\text { A fadiga afetou minha } \\
\text { confiança }\end{array}$ \\
\hline $\begin{array}{l}\text { 26. Fatigue made me feel } \\
\text { unhappy }\end{array}$ & $\begin{array}{l}\text { T1: A fadiga me fez sentir } \\
\text { infeliz } \\
\text { T2: A fadiga me fez sentir } \\
\text { infeliz }\end{array}$ & $\begin{array}{l}\text { A fadiga me fez sentir } \\
\text { infeliz }\end{array}$ & $\begin{array}{l}\text { R1: Fatigue made me unhappy } \\
\text { R2: Fatigue made me feel } \\
\text { unhappy }\end{array}$ & $\begin{array}{l}\text { A fadiga me fez sentir } \\
\text { infeliz }\end{array}$ \\
\hline $\begin{array}{l}27 . \text { I had difficulties sleeping at } \\
\text { night because of fatigue }\end{array}$ & $\begin{array}{l}\text { T1: Tive dificuldade para } \\
\text { dormir à noite por causa } \\
\text { da fadiga } \\
\text { T2: Tive dificuldades para } \\
\text { dormir à noite por causa } \\
\text { da fadiga }\end{array}$ & $\begin{array}{l}\text { Tive dificuldade para } \\
\text { dormir à noite por causa } \\
\text { da fadiga }\end{array}$ & $\begin{array}{l}\text { R1: I had difficulty sleeping at } \\
\text { night because of fatigue } \\
\text { R2: I had difficulty sleeping at } \\
\text { night because of fatigue }\end{array}$ & $\begin{array}{l}\text { Tive dificuldade para } \\
\text { dormir à noite por } \\
\text { causa da fadiga }\end{array}$ \\
\hline $\begin{array}{l}\text { 28. Fatigue affected my ability to } \\
\text { do all my normal household } \\
\text { activities }\end{array}$ & $\begin{array}{l}\text { T1: A fadiga afetou minha } \\
\text { capacidade de realizar } \\
\text { todas as minhas } \\
\text { atividades } \\
\text { domésticas normais } \\
\text { T2: A fadiga afetou minha } \\
\text { capacidade de fazer todas } \\
\text { as minhas atividades } \\
\text { domésticas normais }\end{array}$ & $\begin{array}{l}\text { A fadiga afetou minha } \\
\text { capacidade de fazer } \\
\text { todas as atividades } \\
\text { domésticas normais }\end{array}$ & $\begin{array}{l}\text { R1: Fatigue affected my } \\
\text { capacity to perform all my } \\
\text { normal domestic activities } \\
\text { R2: Fatigue affected my ability } \\
\text { to do all my normal daily } \\
\text { activities }\end{array}$ & $\begin{array}{l}\text { A fadiga afetou minha } \\
\text { capacidade de fazer } \\
\text { todas as atividades } \\
\text { domésticas normais }\end{array}$ \\
\hline $\begin{array}{l}29 . \text { I had to ask others for help } \\
\text { because of fatigue }\end{array}$ & $\begin{array}{l}\text { T1: Precisei pedir ajuda } \\
\text { aos outros por causa da } \\
\text { fadiga } \\
\text { T2: Tive que pedir ajuda } \\
\text { aos outros por causa da } \\
\text { fadiga }\end{array}$ & $\begin{array}{l}\text { Precisei pedir ajuda aos } \\
\text { outros por causa da } \\
\text { fadiga }\end{array}$ & $\begin{array}{l}\mathrm{R} 1 \text { : I needed to ask for help } \\
\text { from others due to fatigue } \\
\mathrm{R} 2 \text { : I needed to ask others for } \\
\text { help because of fatigue }\end{array}$ & $\begin{array}{l}\text { Precisei pedir ajuda } \\
\text { aos outros por causa } \\
\text { da fadiga }\end{array}$ \\
\hline $\begin{array}{l}\text { 30. Quality of my life was affected } \\
\text { by fatigue }\end{array}$ & $\begin{array}{l}\text { T1: A qualidade da minha } \\
\text { vida foi afetada pela fadiga } \\
\text { T2: A qualidade da minha } \\
\text { vida foi afetada pela fadiga }\end{array}$ & $\begin{array}{l}\text { A minha qualidade de } \\
\text { vida foi afetada pela } \\
\text { fadiga }\end{array}$ & $\begin{array}{l}\text { R1: My quality of life was } \\
\text { affected by fatigue } \\
\text { R2: My quality of life was } \\
\text { affected by fatigue }\end{array}$ & $\begin{array}{l}\text { A minha qualidade de } \\
\text { vida foi afetada pela } \\
\text { fadiga }\end{array}$ \\
\hline $\begin{array}{l}\text { SESSÃO III } \\
\text { 1. What do you think is the main } \\
\text { cause of your fatigue apart from } \\
\text { IBD? }\end{array}$ & $\begin{array}{l}\text { T1: } 1 . \text { Na sua opinião, qual } \\
\text { é a principal causa da sua } \\
\text { fadiga, além da DII? } \\
\text { T2: Em sua opinião, qual é } \\
\text { a causa principal da sua } \\
\text { fatiga além da DII? }\end{array}$ & $\begin{array}{l}\text { Na sua opinião, qual é a } \\
\text { principal causa da sua } \\
\text { fadiga, além da DIl? }\end{array}$ & $\begin{array}{l}\text { R1: In your opinion, what is the } \\
\text { principal cause of your fatigue, } \\
\text { in addition to IBD? } \\
\text { R2: In your opinion, what is the } \\
\text { main cause of your fatigue, } \\
\text { aside from IBD? }\end{array}$ & $\begin{array}{l}\text { Na sua opinião, qual é } \\
\text { a principal causa da } \\
\text { sua fadiga, além da } \\
\text { DII? }\end{array}$ \\
\hline $\begin{array}{l}\text { 2. What do you think are the } \\
\text { other causes of your fatigue? }\end{array}$ & $\begin{array}{l}\text { T1: Na sua opinião, quais } \\
\text { são as outras causas da } \\
\text { sua fadiga? } \\
\text { T2: O que você acha que } \\
\text { são as outras causas de } \\
\text { sua fadiga? }\end{array}$ & $\begin{array}{l}\text { Na sua opinião, quais } \\
\text { são as outras causas da } \\
\text { sua fadiga? }\end{array}$ & $\begin{array}{l}\text { R1: In your opinion, what are } \\
\text { the other causes of your } \\
\text { fatigue? } \\
\text { R2: In your opinion, which are } \\
\text { the other causes of your } \\
\text { fatigue? }\end{array}$ & $\begin{array}{l}\text { Na sua opinião, quais } \\
\text { são as outras causas } \\
\text { da sua fadiga? }\end{array}$ \\
\hline $\begin{array}{l}\text { 3. Have you found anything that } \\
\text { helps with your fatigue? }\end{array}$ & $\begin{array}{l}\text { T1: Você já encontrou algo } \\
\text { que ajude a melhorar a } \\
\text { sua fadiga? } \\
\text { T2: Você já encontrou algo } \\
\text { que ajude na sua fadiga? }\end{array}$ & $\begin{array}{l}\text { Você já encontrou algo } \\
\text { que ajude a melhorar a } \\
\text { sua fadiga? }\end{array}$ & $\begin{array}{l}\text { R1: Have you found something } \\
\text { that helps to improve your } \\
\text { fatigue? } \\
\text { R2: Have you already found } \\
\text { something that helped } \\
\text { ameliorate your fatigue? }\end{array}$ & $\begin{array}{l}\text { Você já encontrou algo } \\
\text { que ajude a melhorar a } \\
\text { sua fadiga? }\end{array}$ \\
\hline
\end{tabular}




\begin{tabular}{|c|c|c|c|c|}
\hline $\begin{array}{l}\text { 4. How long have you } \\
\text { experienced fatigue? }\end{array}$ & $\begin{array}{l}\text { T1: Há quanto tempo você } \\
\text { sente fadiga? } \\
\text { T2: Há quanto tempo você } \\
\text { experimenta fadiga? }\end{array}$ & $\begin{array}{l}\text { Há quanto tempo você } \\
\text { sente fadiga? }\end{array}$ & $\begin{array}{l}\text { R1: How long have you } \\
\text { experienced your fatigue? } \\
\text { R2: How long have you felt } \\
\text { fatigue? }\end{array}$ & $\begin{array}{l}\text { Há quanto tempo você } \\
\text { sente fadiga? }\end{array}$ \\
\hline $\begin{array}{l}\text { 5. During this time has your } \\
\text { fatigue been: } \\
\text { a) Constant } \\
\text { b) Intermittent }\end{array}$ & $\begin{array}{l}\text { T1: Durante esse tempo, a } \\
\text { sua fadiga tem sido: } \\
\text { a) Constante } \\
\text { b) Intermitente } \\
\text { T2: Durante este tempo, } \\
\text { sua fadiga tem sido: } \\
\text { a) Constante } \\
\text { b) Não constante }\end{array}$ & $\begin{array}{l}\text { Durante esse tempo, a } \\
\text { sua fadiga tem sido: } \\
\text { a) Constante } \\
\text { b) Não constante }\end{array}$ & 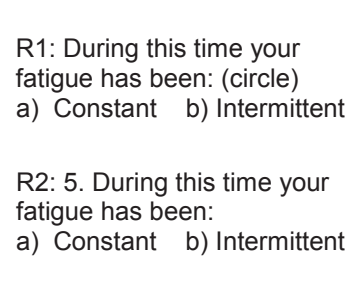 & $\begin{array}{l}\text { Durante esse tempo, a } \\
\text { sua fadiga tem sido: } \\
\text { a) Constante } \\
\text { b) Não constante }\end{array}$ \\
\hline
\end{tabular}

FIGURE 2: end.

TABLE 2. Data of measurement properties testing of the IBD-F Brazil.

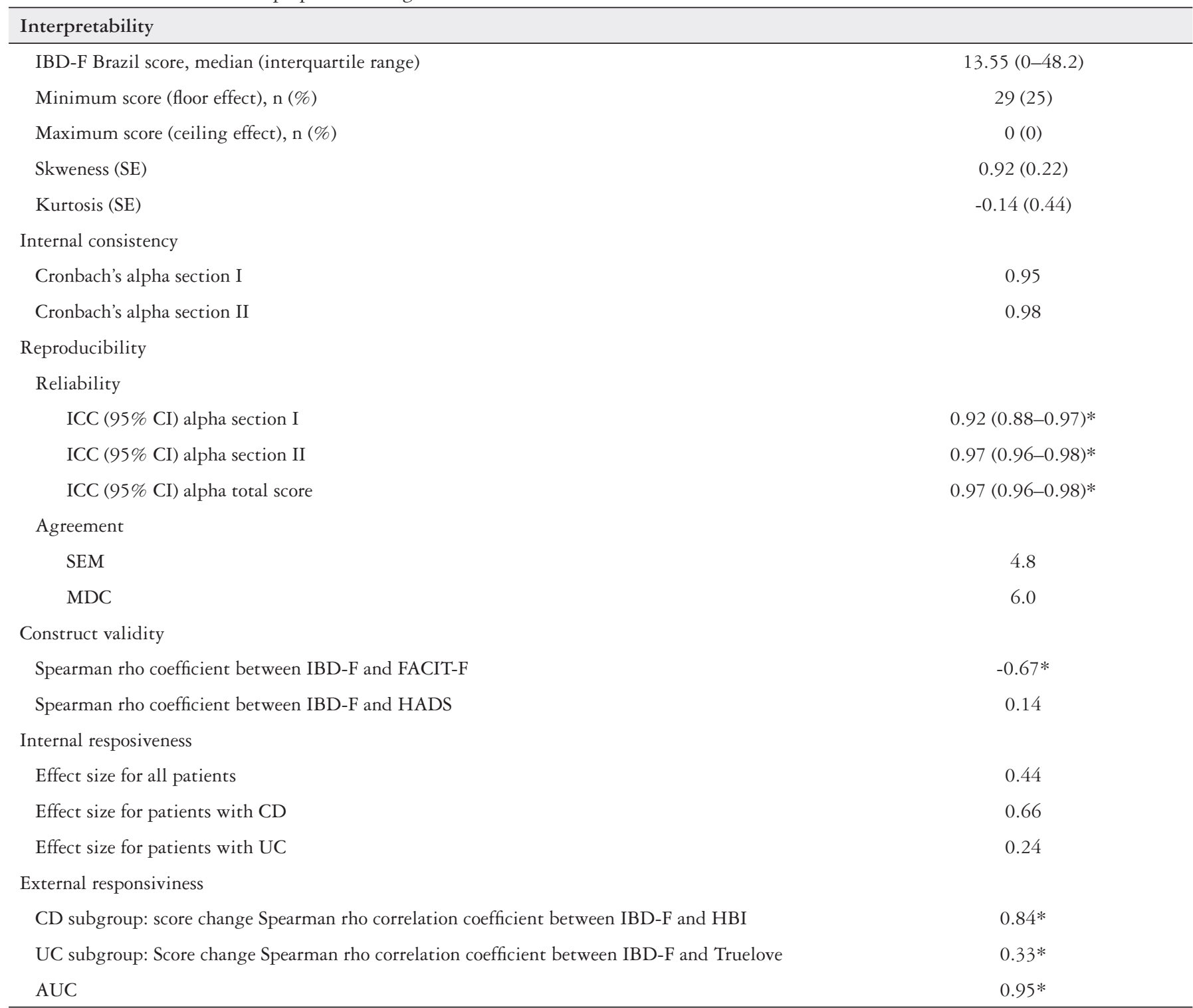

AUC: area under the curve; CD: Crohn's disease; CI: confidence interval; FACIT-F: Functional Assessment of Chronic Illness Therapy-Fatigue; HADS: Hospital Anxiety and Depression Scale; HBI Harvey-Bradshaw index; ICC: intraclass correlation coefficient; MDC: minimal detectable change; SE: standard error; SEM: standard error of the measurement; UC: ulcerative colitis. $* P<0.05$. 
removed. The IBD-F Brazil indicated excellent reliability with ICC ranging from 0.92 in Section I, 0.97 in Section II and 0.97 in the total score. The SEM for the total score was 4.8 and a MDC of 6.05 points. A significant correlation between IBD-F Brazil scale and the FACIT-F were found -0.60 (rho=-0.67; $P<0.001$ ), which confirms the initial hypothesis for convergent validity (TABLE 2). However, no correlation was found between the IBD-F Brazil and the HADS ( $\mathrm{rho}=0.14 ; P=0.12$ ).

The internal responsiveness were analysed at baseline and following 8 to 12 weeks. The effect sizes for all patients in the IBD-F Brazil were lower (0.44). The effect sizes analyzed for subgroups were moderate for patients with Crohn's disease (0.66) and low for patients with ulcerative colitis $(0.24)$. The external responsiveness explored on the correlations between the IBD-F Brazil change scores at 8 to 12 weeks and the Harvey-Bradshaw Index change scores for patients with Crohn's disease were high ( $\mathrm{r}=0.84$, $P<0.001$ ). The correlation between the IBD-F Brazil change scores at 8 to 12 weeks and the Truelove for patients with ulcerative colitis was low ( $\mathrm{r}=0.24, P<0.001)$. The ROC curve indicated a cut-off of 11 points to discriminate between patients with presence of fatigue ( $>11$ points) and absence of fatigue, with a sensitivity $=79.5 \%$, error $=3.7 \%$; specificity $=77 \%$; and an area under the ROC curve $=0.84$ [95\% CI: 0.77-0.92, $P<0.0001]$.

The ceiling and floor effects recorded were $25 \%$ and $0 \%$, respectively. The IBD-F Brazil scores were slightly skewed (Skewness= 0.92 and Kurtosis $=-0.14$ ).

The clinical phase of the disease (activity or remission), for both group of patients with Crohn's disease and ulcerative colitis and the fatigue scores reported in the IBD-F Brazil at different timepoints are presented in TABLE 3 . The fatigue scores were higher in patients with active disease compared to those in the remission phase for Crohn's disease and ulcerative colitis, although patients with Crohn's also disease reported fatigue symptoms during the remission phase.

\section{DISCUSSION}

The main findings of this study were 1) the translational and cross-cultural adaptation Brazilian-Portuguese version of the IBD-F scale and 2) the adequate measurement properties of the IBD-F Brazil to assess fatigue in Brazilian-Portuguese speaking patients. During the translation and cross-cultural adaptation process, only two items from the original version were modified and replaced according to the judgment of the experts committee. The scale was appropriate for and easy to understand by the target population according to legibility scale of Fernandez-Huerta ${ }^{(20)}$. Our study demonstrated that the IBD-F Brazil scale has adequate measurement properties to assess fatigue and its effects on daily living activities in patients with IBD.

The IBD-F Brazil achieved a Cronbach's alpha coefficient of 0.95 and 0.98 for its Section I and for Section II, respectively. These findings were similar to the study of Czuber-Dochan et al. ${ }^{(11)}$ of the original English version of the questionnaire. The high internal consistency indicates that all items from IBD-F adequately measure the fatigue construct. The reproducibility of the IBD-F Brazil questionnaire was excellent (ICC: 0.92 for Section I and 0.97 for Section II) ${ }^{(18)}$, and higher than the one reported by Czuber-Dochan et al (ICC: 0.74 for Section I and 0.83 for Section II). This diverging finding on reability observed between the studies may be explained by the different time points established for test-retest in this study, which may have influenced changes in the perception of fatigue. The measurement error of the instrument of 4.8 points in the IBD-F Brazil was very good ${ }^{(27)}$. The SEM allows identifying how much variation in the score indicates a real change and provides the MDC, which represents the minimum change in score needed to certify that it was not liable to the instrument error. Our results showed that the magnitude of the change required to exceed the variability of the IBD-F Brazil questionnaire is 6.05 points.

TABLE 3. Clinical phase for Crohn's disease ulcerative colitis and IBD-F Brazil scores at different time-points.

\begin{tabular}{|c|c|c|c|c|}
\hline Patient group & Clinical phase & $\begin{array}{c}\text { Baseline } \\
\mathrm{n}=118\end{array}$ & $\begin{array}{c}48 \text { to } 42 \mathrm{~h} \\
\mathrm{n}=118\end{array}$ & $\begin{array}{c}8 \text { to } 12 \mathrm{w} \\
\mathrm{n}=118\end{array}$ \\
\hline \multicolumn{5}{|c|}{ IBD-F score - Section I } \\
\hline \multirow{2}{*}{$\mathrm{CD}$} & Remission $(\mathrm{n}=47)$ & $3.0(0-17)$ & $1.0(0-18)$ & $4.0(0-15)$ \\
\hline & Activity $(\mathrm{n}=34)$ & $13.0(4-18)$ & $13.0(6-18)$ & $4.0(0-12)^{*}$ \\
\hline \multirow{2}{*}{$\mathrm{UC}$} & Remission $(n=26)$ & $2.5(0-16)$ & $0.0(0-12)$ & $0.0(0-17)$ \\
\hline & Activity $(\mathrm{n}=11)$ & $10.5(0-17)$ & $11.5(4-17)$ & $3.0(0-17)^{*}$ \\
\hline \multicolumn{5}{|c|}{ IBD-F score - Section II } \\
\hline \multirow{2}{*}{$\mathrm{CD}$} & Remission ( $\mathrm{n}=47$ ) & $2.0(0-81)$ & $0.0(0-94)$ & $14(0-81)^{*}$ \\
\hline & Activity $(\mathrm{n}=34)$ & $54.5(5-100)$ & $50.0(4-100)$ & $10.5(0-52) *$ \\
\hline \multirow{2}{*}{$\mathrm{UC}$} & Remission ( $\mathrm{n}=26$ ) & $0.0(0-82)$ & $0.0(0-76)$ & $0.0(0-93)$ \\
\hline & Activity $(\mathrm{n}=11)$ & $26.5(0-53)$ & $33.0(4-59)$ & $5.0(0-36)^{*}$ \\
\hline \multicolumn{5}{|l|}{ IBD-F total score } \\
\hline $\mathrm{CD}$ & Activity $(\mathrm{n}=34)$ & $51.0(4-111)$ & $57.3(6-108)$ & $11.0(0-58)^{*}$ \\
\hline \multirow{2}{*}{$\mathrm{UC}$} & Remission ( $\mathrm{n}=26$ ) & $0.0(0-53)$ & $0.0(0-81)$ & $0.0(0-100)$ \\
\hline & Activity $(\mathrm{n}=11)$ & $29.0(0-55)$ & $34.5(4-61)$ & $5.0(0-36) *$ \\
\hline
\end{tabular}

Data are presented as median (min.-max.). CD: Crohn's disease; IBD-F: inflammatory bowel disease- fatigue; UC: ulcerative colitis. $* P<0.01$. 
There was a negative correlation between the IBD-F Brazil and the FACIT-F indicating good content validity. Similar findings were observed between the original English version of IBD-F and instruments used to assess fatigue in a general context ${ }^{(11)}$. The absence of correlation between the IBD-F Brazil and the HADS scale can be explained by the absence of items assessing physical function in the latter, which can considerably contribute to the increase of anxiety and depression among patients with $\mathrm{IBD}^{(29)}$. This finding also brings attention to the combined use of the IBD-F and HADS in clinical practice to assure clinicians of a more thorough assessment of physical and mental health in patients with IBD. The effect size was small in Phase II for the whole group, and moderate among patients with Crohn's disease, which provides evidence of good internal responsiveness of the IBD-F Brazil ${ }^{(28)}$. This difference can be explained by the more severe IBD observed in patients with Crohn's disease who tend o present with more severe symptoms of fatigue due to the varying clinical course of the disease.

The ceiling effect was not detected in the IBD-F Brazil, however, the floor effect was found in $25 \%$ of participants. The majority of the investigated patients were in the remission phase of the disease at the baseline, which may have contributed to the lower prevalence of fatigue reported by these participants, particularly in those with ulcerative colitis. The high prevalence of fatigue observed during the active phase of the disease can be explained by higher levels of blood proinflammatory cytokines such as TNF- $\alpha^{(30)}$, iron deficiency and anemia, a common symptom reported by patients with IBD due to difficulty of iron absorption. In addition, during the active phase of the disease patients with IBD are prone to fatigue induced by drug therapy such as thiopurines and corticotherapy ${ }^{(31)}$.

The cut-off point of 11 in the IBD-F Brazil section I had high sensitivity $(79.5 \%)$ and specificity $(77 \%)$ in detecting fatigue. This cutoff had adequate discriminatory ability, since patients in the active phase of the disease presented with higher scores than those recorded during the remission phase. The identification of a cut-off for the IBD-F Brazil is clinically relevant as it may help to identify patients with IBD who may benefit from an early intervention to reduce fatigue symptom. Fatigue also reduced from baseline to 8 to 12-week follow-up in patients with active Crohn's disease and ulcerative colitis by $69.23 \%$ and $71.43 \%$, respectively. However, fatigue total score increased from baseline to to 8 to 12 -week follow-up in patients with Crohn's disease in those at the remission phase and did not change in patients with ulcerative colitis. The diverging patterns of fatigue over time confirm the varying clinical course between the two IBD diseases. Although the ongoing systemic inflammation plays a central role in the pathogenesis of fatigue in IBD, the prevalence of fatigue among patients with IBD during clinical remission was $40 \%{ }^{(32)}$.
This study has limitations. First, the recruitment of participants with IBD was conducted from single center, however, the participant's characteristics were similar in age and disease duration reported in previous studies also carried out in the general IBD population $^{(4,6,7)}$. Second, the IBD-F was originally self-administered by the study participants. In this study the IBD-F Brazil was evaluated by interview, further studies are required to explore the feasibility of a self-administered version. Finally, an objective measure of muscle fatigue was not used. The associations between the IBD-F Brazil and objective measures of fatigue warrant investigation in future studies.

\section{CONCLUSION}

The Brazilian-Portuguese versions of the IBD-F has adequate measurement properties to assess fatigue and its effects on activities of daily living of patients with IBD, particularly in Crohn's disease. The IBD-F Brazil should be included as part of the clinical assessment of patients with IBD. These findings suggest that the IBD-F Brazil can be used in clinical practice and research.

\section{Authors' contribution}

Lage AC: acquisition of data, analysis and interpretation of data, drafting the article, final approval of the version to be submitted. Oliveira CC: conception and design of the study, analysis and interpretation of data, drafting the article, final approval of the version to be submitted. Batalha APDB: acquisition of data, revising the article critically for important intellectual content, final approval of the version to be submitted. Araújo AF: acquisition of data, revising the article critically for important intellectual content, final approval of the version to be submitted. Czuber-Dochan W: conception and design of the study, analysis and interpretation of data, drafting the article, final approval of the submitted version. JMF Chebli: conception and design of the study, analysis and interpretation of data, drafting the article, final approval of the version to be submitted. Cabral LA: acquisition of data, revising the article critically for important intellectual content, final approval of the version to be submitted. C Malaguti: conception and design of the study, analysis and interpretation of data, drafting the article, final approval of the version to be submitted.

\section{Orcid}

Ana Cristina Lage: 0000-0003-1993-0438.

Cristino Carneiro Oliveira: 0000-0001-6546-0225.

Ana Paula Delgado Bomtempo Batalha: 0000-0003-2938-3116.

Adaliza Furtado Araújo: 0000.0002.4901.8995.

Wladyslawa Czuber-Dochan: 0000.0002.9889.1248

Júlio Maria Fonseca Chebli: 0000.0003.1527.0663.

Laura Alves Cabral: 0000.0002.8315.4114.

Carla Malaguti: 0000.0002.6619.136X. 
Lage AC, Oliveira CC, Batalha APDB, Araújo AF, Czuber-Dochan W, Chebli JMF, Cabral LA, Malaguti C. Escala de autoavaliação Inflammatory Bowel Disease-Fatigue: tradução, adaptação cultural e propriedades psicométricas da versão Brasileira (IBD-F Brasil). Arq Gastroenterol. 2020;57(1):50-63.

RESUMO - Contexto - A fadiga é um sintoma comum em pacientes com doenças inflamatórias intestinais (DII). Um instrumento de avaliação de fadiga traduzido, culturalmente adaptado e com psicometria robusta para medir a fadiga em pacientes brasileiros com DII é necessário. Objetivo - Traduzir e adaptar culturalmente a Inflammatory Bowel Disease Fatigue Scale (IBD-F) para o português do Brasil e testar suas propriedades de medida em pacientes brasileiros com DII. Métodos - Foram coletados dados de 123 pacientes com DII. Além do IBD-F, foi utilizada a Functional Assessment of Chronic Illness Therapy-Fatigue (FACIT-F). As propriedades de medida testadas foram: consistência interna, reprodutibilidade (confiabilidade e concordância), validade de construto, responsividade interna e externa e efeitos teto e chão. Resultados - A versão em português do IBD-F mostrou excelente consistência interna (alfa de Cronbach de 0,95), excelente reprodutibilidade (ICC=0,97) e uma diferença mínima detectável de 6,0 pontos. A validade do construto foi demonstrada por meio de uma boa correlação entre o IBD-F Brasil e o FACIT-F ( $\mathrm{r}=-0,46)$. Na análise de responsividade interna, os tamanhos de efeito obtidos foram moderado entre aqueles com doença de Crohn $(0,66)$ e baixo em pacientes com colite ulcerativa $(0,24)$. O IBD-F Brasil apresentou alta responsividade externa entre aqueles com doença de Crohn $(0,84)$ e baixa responsividade externa em pacientes colite ulcerativa $(0,33)$. A área sob a curva considerada para responsividade foi de 0,84 . Foram registrados $25 \%$ de efeito chão e nenhum efeito teto nas avaliações realizadas. Conclusão - O IBD-F Brasil possui propriedades de medida adequadas e seu uso pode ser recomendado na prática clínica e na pesquisa em pacientes com DII.

DESCRITORES - Doenças inflamatórias intestinais. Reprodutibilidade dos testes. Fadiga.

\section{REFERENCES}

1. Loftus EV. Clinical epidemiology of inflammatory bowel disease: incidence, prevalence, and environmental influences. Gastroenterology. 2004;126:1504-17.

2. Kreijne JE, Lie MRKL, Vogelaar L, van der Woude CJ. Practical Guideline for Fatigue Management in Inflammatory Bowel Disease. J Crohns Colitis. 2015;10:105-11.

3. Lichtenstein GR, Hanauer SB, Sandborn WJ. Management of Crohn's Disease in Adults. Am J Gastroenterol. 2009;104:465-83.

4. Langenberg DR, Gibson PR. Systematic review: fatigue in inflammatory bowel disease. Aliment Pharmacol Ther. 2010;32:131-43.

5. Cella D, Yount S, Rothrock N, Gershon R, Cook K, Reeve B, et al. PROMIS Cooperative Group. The Patient-Reported Outcomes Measurement Information System (PROMIS) Progress of an NIH Roadmap Cooperative Group During its First Two Years. Med Care. 2007;45(5 Suppl 1): S3-11.

6. Matura LA, Malone S, Jaime-Lara R, Riegel B. A Systematic Review of Biological Mechanisms of Fatigue in Chronic Illness. Biol Res Nurs. 2018;20:410-21.

7. Romberg-Camps MJ, Bol Y, Dagnelie PC, Hesselink-van de Kruijs MA, Kester AD, Engels LG, et al. Fatigue and Health-related Quality of Life in Inflammatory Bowel Disease: Results from a Population-Based Study in the Netherlands: The IBD-South Limburg Cohort. Inflamm Bowel Dis. 2010;16:2137-47.

8. Czuber-Dochan W, Ream E, Norton C. Review article: description and management of fatigue in inflammatory bowel disease. Aliment Pharmacol Ther. 2013:37:505-16.

9. Tinsley A, Macklin EA, Korzenik JR, Sands BE. Validation of the Functional Assessment of Illness Therapy-Fatigue (FACIT-F) in patients with inflammatory bowel disease. Aliment Pharmacol Ther. 2011;34:1328-36.

10. Czuber-Dochan W, Norton C, Bassett P, Berliner S4, Bredin F5, Darvell M, et al. Development and psychometric testing of inflammatory bowel disease fatigue (IBD-F) patient self-assessment scale. J Crohns Colitis. 2014;8:1398-406.

11. Artom M, Czuber-Dochan W, Sturt J, Murrells T, Norton C. The contribution of clinical and psychosocial factors to fatigue in 182 patients with inflammatory bowel disease: a cross-sectional study. Aliment Pharmacol Ther. 2017;45:403-16.

12. Artom M, Czuber-Dochan W, Sturt J, Norton C. Cognitive behavioural therapy for the management of inflammatory bowel disease-fatigue with a nested qualitative element: study protocol for a randomized controlled trial. Trials. 2017; $18: 213$.

13. Tew GA, Jones K, Mikocka-Walus A. Physical Activity Habits, Limitations, and Predictors in People with Inflammatory Bowel Disease: A Large Cross-sectional Online Survey. Inflamm Bowel Dis. 2016;22:2933-42.

14. Tew GA, Carpenter R, Seed M, Anderson S, Langmead L, Fairhurst C, Bottoms L. Feasibility of high-intensity interval training and moderate-intensity continuous training in adults with inactive or mildly active Crohn's disease: study protocol for a randomised controlled trial. Pilot Feasibility Study. 2017;3:17.

15. Mokkink LB, Terwee CB, Patrick DL, Alonso J, Stratford PW, Knol DL, et al. The COSMIN study reached international consensus on taxonomy, terminology, and definitions of measurement properties for health-related patient-reported outcomes. J Clin Epidemiol. 2010;63:737-45.
16. Gasparini RG. Incidência e Prevalência de Doenças Inflamatórias Intestinais no Estado de São Paulo-Brasil.2018;78-79. [Accessed 2018 May 1]. Available at https://repositorio.unesp.br/bitstream/handle/11449/152905/gasparini_rg_dr_bot. pdf? sequence $=3$.

17. Terwee CB, Bot SDM, de Boer MR, van der Windt DA, Knol DL, Dekker J, et al. Quality criteria were proposed for measurement properties of health status questionnaires. J Clin Epidemiol. 2007;60:34-42.

18. Fernández Huerta J. Medidas sencillas de lecturabilidad. Consigna. 1959;214:29-32.

19. Beaton DE, Bombardier C, Guillemin F, Ferraz MB. Guidelines for the process of cross-cultural adaptation of self-report measures. Spine. 2000;25:3186-91.

20. Brasil. Ministério da Saúde. Protocolo Clínico e Diretrizes Terapêuticas Doença de Crohn. Portaria SAS/MS n ${ }^{\circ}$ 966, de 2 de outubro de 2014.

21. Brasil. Ministério da Saúde. Protocolo Clínico e Diretrizes Terapêuticas Retocolite Ulcerativa. Portaria SAS/MS n ${ }^{\circ} 861$, de 4 de novembro de 2002.

22. Harvey RF, Bradshaw, JM. A simple index of Crohn's-disease activity. Lancet. 1980;1:514.

23. Truelove SC, Witts LJ. Cortisone in ulcerative colitis final report on a therapeutic trial. Br Med J. 1955;2:1041-8.

24. Elliott PR, Leonnard-Jones JE, Hathway N. Simple Index of Crohn's Disease Activity. Lancet 1980;1:876.

25. Acaster S, Dickerhoof R, DeBusk K, Bernard K, Strauss W, Allen LF. Qualitative and quantitative validation of the FACIT-fatigue scale in iron deficiency anemia. Health Qual Life Outcomes. 2015;13:60-70.

26. Pais-Ribeiro J, Silva I, Ferreira T, Martins A, Meneses R, Baltar M. Validation study of a Portuguese version of the Hospital Anxiety and Depression Scale. Psychol Health Med. 2007;12:225-37.

27. Portney LG, Watkins MP. Foundations of Clinical Research: Applications to Practice, 2nd ed. Upper Sadde River: Prentice-Hall, 2000.

28. Husted JA, Cook RJ, Farewell VT, Gladman DD. Methods for assessing responsiveness: a critical review and recommendations. J Clin Epidemiol. 2000;53:459-68.

29. Bjelland I, Dahl AA, Haug TT, Neckelmann D. The validity of the Hospital Anxiety and Depression Scale An updated literature review. J Psychosom Res. 2002;52:69-77.

30. Dave M, Papadakiska, Faubion WA Jr. Immunology of Inflammatory Bowel Disease and Molecular Targets for Biologics. Gastroenterol Clin North Am. 2014:43:405-24.

31. Minderhoud IM, Samsom M, Oldenburg B. Crohn's disease, fatigue, and infliximab: Is there a role for cytokines in the pathogenesis of fatigue? World $\mathbf{J}$ Gastroenterol 2007;13:2089-93.

32. Bager P, Befrits R, Wikman O, Lindgren S, Moum B, Hjortswang H, Hjollund NH, Dahlerup JF. Fatigue in out-patients with inflammatory bowel disease is common and multifactorial. Aliment Pharmacol Ther. 2012;35:133-41. 